Research Article

\title{
Some Methods about Finding the Exact Solutions of Nonlinear Modified BBM Equation
}

\author{
Fan Niu $\left(\mathbb{D},{ }^{1}\right.$ Jianming Qi $\mathbb{D}^{1},{ }^{1}$ and Zhiyong Zhou $\mathbb{D}^{2,3}$ \\ ${ }^{1}$ School of Business, Shanghai Dianji University, Shanghai 201306, China \\ ${ }^{2}$ School of Biomedical Engineering, Shanghai Jiaotong University, Shanghai 200240, China \\ ${ }^{3}$ School of Design and Art, Shanghai Dianji University, Shanghai 201306, China \\ Correspondence should be addressed to Zhiyong Zhou; zhouzhiyong001@126.com
}

Received 24 January 2021; Revised 20 February 2021; Accepted 18 March 2021; Published 7 April 2021

Academic Editor: Mostafa M. A. Khater

Copyright ( $\odot 2021$ Fan Niu et al. This is an open access article distributed under the Creative Commons Attribution License, which permits unrestricted use, distribution, and reproduction in any medium, provided the original work is properly cited.

\begin{abstract}
Finding exact solutions of nonlinear equations plays an important role in nonlinear science, especially in engineering and mathematical physics. In this paper, we employed the complex method to get eight exact solutions of the modified BBM equation for the first time, including two elliptic function solutions, two simply periodic solutions, and four rational function solutions. We used the $\exp (-\phi(z))$-expansion methods to get fourteen forms of solutions of the modified BBM equation. We also used the sinecosine method to obtain eight styles' exact solutions of the modified BBM equation. Only the complex method can obtain elliptic function solutions. We believe that the complex method presented in this paper can be more effectively applied to seek solutions of other nonlinear evolution equations.
\end{abstract}

\section{Introduction}

It is well known that modern natural science is undergoing profound changes. Nonlinear science runs through the fields of mathematical science, life science, space science, and electrical engineering [1] and has become an important frontier of contemporary scientific research. In the field of electrical engineering, the security of distribution network systems reliability and economy involve many nonlinear sciences [2-4]. The development of nonlinear sciences provides more accurate reference information for power system operation and scheduling [5] and also promotes the development of nonlinear sensitive electronic devices, which establish the foundation for the extensive use of nonlinear electrical equipment on the load side and grid side of the power system [6,7], such as the nonlinear controller in the differential model of the integrated control system of the generator, which improves the standard and transient stability of the generator excitation system in the power system [8]. Therefore, constructing a more accurate nonlinear mathematical model in the power system and solving the exact solution of the equations in the model are of great significance to the analysis of the power system [9-11].

The exact solutions of nonlinear partial differential equations have always attracted much attention; many effective methods for solving nonlinear partial differential equations have been continuously proposed and improved $[12,13]$. Gardner et al. [14] proposed the nonlinear Fourier transform method. Lou and Tang [15] established and improved the multilinear separation variable method to solve a large class of $(2+1)$-dimensional nonlinear systems. The projection Riccati equation expansion method was proposed by Musette and Conte [16]. He and $\mathrm{Wu}$ [17] proposed the exponential function method. Khater et al. [18-20] also had made the outstanding and huge contribution in related fields. Many scholars later proposed other methods, such as direct algebra method [21], tanh function expansion method [22], and Weierstrass elliptic function expansion method [23]. There are also methods based on symbolic calculations for solitary wave solutions of nonlinear partial differential equations [24, 25].

In 1972, Benjamin-Bona-Mahony equation was introduced which is a nonlinear partial differential equation with 
small amplitude and long wave in the simulation of fluid mechanics by Benjamin, Bona, and Mahony [26]. One of the important models of long-wave one-way propagation in weakly nonlinear dispersive media is the BBM equation [27], later developed into mBBM. In 1991, Zhang [28] used the undetermined coefficient method to construct bell-shaped and twisted solitary wave solutions. In 1998, Shang [29] used direct and hypothetical methods to find the twisted and antitwisted solitary wave solution clocks, shaped solitary wave solutions, trigonometric function waves, and singular traveling wave solutions for BBM and modified BBM equations. In 2003, Liu [30] constructed the exact solution of the BBM equation by using the Jacobi elliptic function expansion method. In 2004, Tautusan [31] constructed an exact solitary wave solution of the BBM equation and the mBBM equation by combining auxiliary equations and hyperbolic function assumptions. In 2013, Liu [32] used Painlevé direct truncation method to find the exact solution of mBBM equation. In 2017, Yang and Feng [33] applied the improved simple equation method to find the exact solution of the mBBM equation. In 2020, Gupta [34] generalised Kudryashov technique which has been implemented to construct new solutions of modified BBM equation. In this paper, we used the complex method $[35,36]$ and the $\exp (-\phi(z))$-expansion method [37] and sine-cosine method [38] to find the exact solutions of modified Benjamin-BonaMahony equation.

Modified BBM equation (see $[39,40]$ ) is given as

$$
u_{t}+u_{x}+u^{2} u_{x}+\beta u_{x x t}=0 .
$$

In (1), $\beta$ is constant. By putting

$$
\begin{aligned}
u(x, t) & =w(z), \\
z & =k(x-\lambda t),
\end{aligned}
$$

into (1), and integrating it deduces

$$
\beta \lambda k^{2} w^{\prime \prime}-\frac{1}{3} w^{3}+(\lambda-1) w-b=0 .
$$

In order to get the exact solution of mBBM equation, we employ complex method to seek for the exact solution of (3), and we also use the $\exp (-\phi(z))$-expansion method to get some exact solutions and use sine-cosine method to find the exact solutions of modified Benjamin-Bona-Mahony equation.

\section{Introduction of Complex Method and Some Lemmas and Main Result}

In order to give our complex method, we have to know some concepts and symbols. First, we set $m \in \mathbb{N}:=\{1,2,3, \ldots$,$\} ,$ $r_{j} \in \mathbb{N}_{0}=\mathbb{N} \cup\{0\}, r=\left(r_{0}, r_{1}, \ldots, r_{m}\right), j=0,1, \ldots, m$. Then, we can get a differential monomial by

$$
\begin{aligned}
M_{r}[w](z):= & {[w(z)]^{r_{0}}\left[w^{\prime}(z)\right]^{r_{1}} } \\
& \cdot\left[w^{\prime \prime}(z)\right]^{r_{2}}, \ldots,\left[w^{(m)}(z)\right]^{r_{m}},
\end{aligned}
$$

$p(r):=r_{0}+2 r_{1}+\cdots+(m+1) r_{m}$, and $\operatorname{deg}(M)$ are regarded as the weight and degree of $M_{r}[w]$, separately.

The differential polynomial $P\left(w, w^{\prime}, \ldots, w^{(m)}\right)$ can be defined as follows:

$$
P\left(w, w^{\prime}, \ldots, w^{(m)}\right):=\sum_{r \in I} a_{r} M_{r}[w] .
$$

In equation (5), $a_{r}$ are constants, and $I$ is a finite index set.

The total weight and degree of $P\left(w, w^{\prime}, \ldots, w^{(m)}\right)$ are marked as $W(P):=\max _{r \in I}\{p(r)\}$ and $\operatorname{deg}(P):=\max _{r \in I}$ $\left\{\operatorname{deg}\left(M_{r}\right)\right\}$, separately.

Considering the complex ordinary differential equations,

$$
P\left(w, w^{\prime}, \ldots, w^{(m)}\right)=b w^{n}+c .
$$

In equation (6), $b \neq 0, c$ are constants, $n \in \mathbb{N}$.

We take $p, q \in \mathbb{N}$, and we consider that the meromorphic solutions $w$ of equation (6) have one or more poles. We can get that equation (6) satisfies the $\langle p, q\rangle$ condition, where $p$ indicates that the equation has $p$ distinct meromorphic solutions and $q$ indicates that their multiplicity of the pole at $z=0$ is $q$.

We have a hard time finding the $\langle p, q\rangle$ condition of equation (6), so we need a way to find the weak $\langle p, q\rangle$ condition shown as follows.

To find out the weak $\langle p, q\rangle$ condition of equation (6), we can substitute Laurent series

$$
w(z)=\sum_{k=-q}^{\infty} c_{k} z^{k}, \quad q>0, c_{-q} \neq 0,
$$

into equation (6); then, we can find out the $p$ distinct Laurent singular parts as follows:

$$
\sum_{k=-q}^{-1} c_{k} z^{k}
$$

Given two complex numbers $\omega_{1}, \omega_{2}$, and $\operatorname{Im}\left(\omega_{1} / \omega_{2}\right)>0$, $L=L\left[2 \omega_{1}, 2 \omega_{2}\right]$ are discrete subset $L\left[2 \omega_{1}, 2 \omega_{2}\right]=\{\omega \mid \omega=$ $\left.2 n \omega_{1}+2 m \omega_{2}, n, m \in \mathbb{Z}\right\}$, which is isomorphic to $\mathbb{Z} \times \mathbb{Z}$. Let the discriminant $\Delta=\Delta\left(c_{1}, c_{2}\right):=c_{1}^{3}-27 c_{2}^{2}$ and

$$
s_{n}=s_{n}(L):=\sum_{\omega \in L \backslash\{0\}} \frac{1}{\omega^{n}} .
$$

A meromorphic function $w(z)$ means that $w(z)$ is holomorphic in the complex plane $\mathbb{C}$ except for poles. $\wp\left(z, g_{2}, g_{3}\right)$ is the Weierstrass elliptic function with invariants $g_{2}$ and $g_{3}$.

If $f$ is an elliptic function, or a rational function of $e^{\alpha z}, \alpha \in \mathbb{C}$, or a rational function of $z$, the meromorphic function $f$ belongs to the class $W$.

Weierstrass elliptic function $\wp(z):=\wp\left(z, g_{2}, g_{3}\right)$ is a meromorphic function with double periods $\omega_{1}, \omega_{2}$ defined as 


$$
\wp\left(z ; w_{1}, w_{2}\right):=\frac{1}{z^{2}}+\sum_{\mu, \nu \in \mathbf{Z}, \mu^{2}+\nu^{2} \neq 0}\left\{\frac{1}{\left(z+\mu w_{1}+\nu w_{2}\right)^{2}}-\frac{1}{\left(\mu w_{1}+\nu w_{2}\right)^{2}}\right\}
$$

which satisfies the following equation:

$$
\left(\wp^{\prime}(z)\right)^{2}=4 \wp(z)^{3}-g_{2} \wp(z)-g_{3},
$$

where $g_{2}=60 s_{4}, g_{3}=140 s_{6}$, and $\Delta\left(g_{2}, g_{3}\right) \neq 0$.

Or alternating equation (11) to the form

$$
\left(\wp^{\prime}(z)\right)^{2}=4\left(\wp(z)-e_{1}\right)\left(\wp(z)-e_{2}\right)\left(\wp(z)-e_{2}\right),
$$

where $e_{1}=\wp\left(\omega_{1}\right), e_{2}=\wp\left(\omega_{2}\right), e_{3}=\wp\left(\omega_{1}+\omega_{2}\right)$.

Contrarily, given two complex numbers $g_{2}$ and $g_{3}$ and $\Delta\left(g_{2}, g_{3}\right) \neq 0$, then there will be double periods $\omega_{1}, \omega_{2}$ Weierstrass elliptic function $\wp(z)$ which the solutions will possess.

In 2009, A. E. Eremenko et al. [41] investigated the $m$-order Briot-Bouquet equation (BBEq) as follows:

$$
F\left(w, w^{(m)}\right)=\sum_{j=0}^{m} F_{j}(w)\left(w^{(m)}\right)^{j}=0 .
$$

In equation (13), $F_{j}(w)$ are constant coefficients polynomials, $m \in \mathbb{N}$. There are the following lemmas of the $m$ order BBEq.

Recently, Yuan et al. [35, 36, 39, 42] summarized the work of Eremenko et al. and introduced the complex method to find the exact solutions of nonlinear evolution equations in mathematical physics for the first time. Lemmas 1 and 2 play an important role in the complex method.

Lemma 1 (see [43-45]). Let $p, l, m, n \in \mathbb{N}, \operatorname{deg} P\left(w, w^{(m)}\right)$ $<n$. Consider that an $m$-order Briot-Bouquet equation

$$
P\left(w^{(m)}, w\right)=b w^{n}+c
$$

satisfies weak $\langle p, q\rangle$ condition; then, all the meromorphic solutions $w$ will belong to the class $W$. For some values of parameters, if the solution $w$ exists, then other meromorphic solutions form a one-parametric family $w\left(z-z_{0}\right), z_{0} \in \mathbb{C}$. And then each solution can be written as the following forms with pole at $z=0$ :

$$
\begin{aligned}
w(z)= & \sum_{i=1}^{l-1} \sum_{j=2}^{q_{i}} \frac{(-1)^{j} c_{-i j}}{(j-1) !} \frac{\mathrm{d}^{j-2}}{\mathrm{~d} z^{j-2}}\left(\frac{1}{4}\left[\frac{\wp^{\prime}(z)+B_{i}}{\wp(z)-A_{i}}\right]^{2}-\wp(z)\right) \\
& +\sum_{i=1}^{l-1} \frac{c_{-i 1}}{2} \frac{\wp^{\prime}(z)+B_{i}}{\wp(z)-A_{i}}+\sum_{j=2}^{q_{l}} \frac{(-1)^{j} c_{-l j}}{(j-1) !} \frac{\mathrm{d}^{j-2}}{\mathrm{~d} z^{j-2}} \wp(z)+c_{0} .
\end{aligned}
$$

In equation (15), $c_{-i j}$ are given by equation (5), and $B_{i}^{2}=4 A_{i}^{3}-g_{2} A_{i}-g_{3}, \sum_{i=1}^{l} c_{-i 1}=0$.

Each rational function solution $w:=R(z)$ can be shown as follows:

$$
R(z)=\sum_{i=1}^{l} \sum_{j=1}^{q} \frac{c_{i j}}{\left(z-z_{i}\right)^{j}}+c_{0}
$$

with $l(\leq p)$ distinct poles of multiplicity $q$.

Every simply periodic solution is a rational function $R(\xi)$ of $\xi=e^{\alpha z}(\alpha \in \mathbb{C}) . R(\xi)$ has $l(\leq p)$ distinct poles of multiplicity $q$ and can be shown as follows:

$$
R(\xi)=\sum_{i=1}^{l} \sum_{j=1}^{q} \frac{c_{i j}}{\left(\xi-\xi_{i}\right)^{j}}+c_{0} .
$$

Lemma 2 (see $[45,46])$. The Weierstrass elliptic functions $\wp(z):=\wp\left(z, g_{2}, g_{3}\right)$ have two successive degeneracies and addition formula:

(i) Degeneracy to simply periodic functions (i.e., rational functions of one exponential $e^{k z}$ ) according to

$$
\wp\left(z, 3 d^{2},-d^{3}\right)=2 d-\frac{3 d}{2} \operatorname{coth}^{2} \sqrt{\frac{3 d}{2}} z
$$

if one root $e_{j}$ is double $\left(\left(\Delta\left(g_{2}, g_{3}\right)=0\right)\right)$.

(ii) Degeneracy to rational functions of $z$ according to

$$
\wp(z, 0,0)=\frac{1}{z^{2}}
$$

if one root $e_{j}$ is triple $\left(\left(g_{2}=g_{3}=0\right)\right)$.

(ii) Addition formula

$$
\wp\left(z-z_{0}\right)=-\wp(z)-\wp\left(z_{0}\right)+\frac{1}{4}\left[\frac{\wp^{\prime}(z)+\wp^{\prime}\left(z_{0}\right)}{\wp(z)-\wp\left(z_{0}\right)}\right]^{2} .
$$

Through the above lemma and results, we can introduce complex method to find exact solutions of some PDEs. Here are the five steps:

(1) Substitute the transform $T: u(x, t) \longrightarrow w(z)$, $(x, t) \longrightarrow z$ into a given PDE to produce a nonlinear ODE (6) or (8)

(2) Put equation (7) or (8) into equation (6) to find out weak $\langle p, q\rangle$ condition

(3) By determinant relation equations (15)-(17), we, respectively, find the elliptic, rational, and simply periodic solutions $w(z)$ of equation (6) or (8) with pole at $z=0$

(4) By Lemmas 1 and 2, we can get meromorphic solutions and the addition formula

(5) Put the inverse transform $T^{-1}$ into the meromorphic solutions $w\left(z-z_{0}\right)$, we can obtain all exact solutions $u(x, t)$ of the original PDE 
Theorem 1. When we employ complex method, we suppose $-(1 / 3) \beta \lambda k^{2} \neq 0$; then, all meromorphic solutions $w$ of (3) belong to the class $W$. And we found that (3) has the following three forms of solutions: (a) The elliptic function solutions:

$$
w_{d}(z)= \pm \frac{1}{2} \sqrt{6 \beta \lambda k^{2}} \frac{(-\wp+c)\left(4 \wp c^{2}+4 \wp^{2} c+2 \wp^{\prime} d-\wp g_{2}-c g_{2}\right)}{\left(\left(12 c^{2}-g_{2}\right) \wp+4 c^{3}-3 c g_{2}\right) \wp^{\prime}+\left(4 \wp^{3}+12 c \wp^{2}-3 g_{2} \wp-c g_{2}\right) d}
$$

where, $g_{3}=0, d^{2}=4 c^{3}-g_{2} c, g_{2}$ and $c$ are arbitrary constants.

(b) The simply periodic solutions:

$$
w_{s, 1}(z)=\alpha \sqrt{\frac{3}{2} \beta \lambda k^{2}}\left(\operatorname{coth} \frac{\alpha}{2}\left(z-z_{0}\right)-\operatorname{coth} \frac{\alpha}{2}\left(z-z_{0}-z_{1}\right)-\operatorname{coth} \frac{\alpha}{2} z_{1}\right)
$$

(i) In order to better describe $w_{s, 1}(z)$, we make a graph of the function solution under specific parameters, as shown in Figure 1 in Section 7 and

$$
w_{s, 2}(z)=\alpha \sqrt{\frac{3}{2} \beta \lambda k^{2}}\left(\tanh \frac{\alpha}{2}\left(z-z_{0}\right)\right),
$$

where $z_{0} \in \mathbb{C}, \lambda-1=\beta \lambda k^{2} \alpha^{2}\left((1 / 2)+\left(3 / 2 \sinh ^{2} \quad(\alpha /\right.\right.$ 2) $\left.\left.z_{1}\right)\right), b=-\sqrt{(3 / 2) \beta \lambda k^{2}} \quad\left(\left(\tanh (\alpha / 2) z_{1}\right) /\left(\sinh ^{2}(\alpha /\right.\right.$ 2) $\left.\left.z_{1}\right)\right), z_{1} \neq 0$ in the former formula, or $\lambda-1=$ $\left(\left(\beta \lambda k^{2} \alpha^{2}\right) / 2\right), b=0$.

(c) The rational function solutions:

$$
w_{r, 1}(z)= \pm \sqrt{6 \beta \lambda k^{2}} \frac{1}{z-z_{0}} .
$$

(i) In order to better describe $w_{r, 1}(z)$, we make a graph of the function solution under specific parameters, as shown in Figure 2 in Section 7 and

$w_{r, 2}(z)= \pm \sqrt{6 \beta \lambda k^{2} \frac{1}{z_{1}^{2}}}\left(\frac{z_{1}}{z-z_{0}}-\frac{z_{1}}{z-z_{0}-z_{1}}-1\right)$

where $z_{0} \in \mathbb{C}, \lambda=1, b=0$ in the former formula, or given $\quad z_{1} \neq 0, \lambda-1=\left(\left(6 \beta \lambda k^{2}\right) / z_{1}^{2}\right),-b=\mp(2 / 3)$ $\left(\left(6 \beta \lambda k^{2}\right) / z_{1}^{2}\right)^{(3 / 2)}$.

\section{Introduction of $\operatorname{Exp}(-\phi(z))$-Expansion Method and Main Result}

Consider the following form of a nonlinear partial differential equation (PDE):

$$
P\left(\mu, \mu_{x}, \mu_{t}, \mu_{x x}, \mu_{t t}, \ldots,\right)=0,
$$

where $P$ is a polynomial with an unknown function $\mu(x, y, t)$ and its derivatives in which nonlinear terms and highest order derivatives are involved. And it can be processed as follows:
Step 1. Inserting the traveling wave transform $\mu(x, t)=w(z), z=k(x-\lambda t)$ into equation (26), alternating it to the following ordinary differential equation (ODE):

$$
K\left(w, w^{\prime}, w^{\prime \prime}, w^{\prime \prime \prime}, \ldots,\right)=0
$$

where $K$ is a polynomial of $w(z)$ and its derivatives. Step 2. Regarding that equation (27) has the following traveling wave solution:

$$
w(z)=\sum_{j=0}^{n} C_{j}(\exp (-\phi(z)))^{j}
$$

where $C_{j}(0 \leq j \leq n)$ are constants and will be determined later, and $C_{j} \neq 0$ and $\phi=\phi(z)$ satisfies the ODE as follows:

$$
\phi^{\prime}(z)=\exp (-\phi(z))+\mu \exp (\phi(z))+\delta .
$$

(i) Equation (29) has different style solutions as follows: If $\delta^{2}-4 \mu>0, \mu \neq 0$, then

$\phi(z)=\ln \left(\frac{-\sqrt{\left(\delta^{2}-4 \mu\right)} \tanh \left(\left(\sqrt{\left(\delta^{2}-4 \mu\right)} / 2\right)(z+c)-\delta\right)}{2 \mu}\right)$,

$$
\phi(z)=\ln \left(\frac{-\sqrt{\left(\delta^{2}-4 \mu\right)} \operatorname{coth}\left(\left(\sqrt{\left(\delta^{2}-4 \mu\right)} / 2\right)(z+c)-\delta\right)}{2 \mu}\right)
$$

If $\delta^{2}-4 \mu<0, \mu \neq 0$, then 


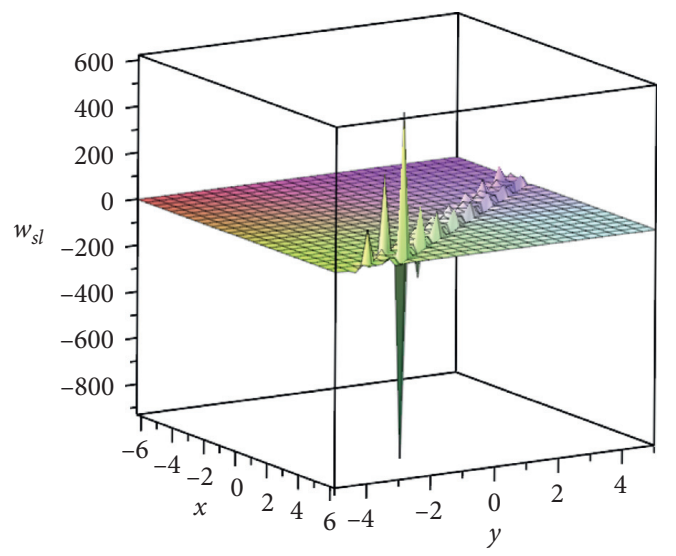

FIGURE 1: The solution of $\mathrm{mBBM}$ equation corresponding to $w_{s, 1}(z), \quad$ take $\quad \alpha=2, \quad \beta=\left(1 /\left(3\left[1+3(\operatorname{csch} 1)^{2}\right]\right)\right), \quad \lambda=3$, $k=1, z_{0}=2, z_{1}=1$.

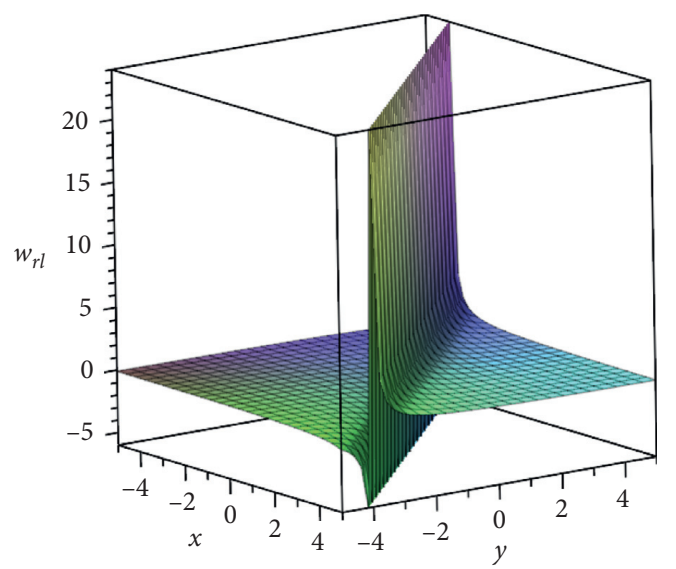

FIgURE 2: The solution of $\mathrm{mBBM}$ equation corresponding to $w_{r, 1}(z)$, take $\beta=(1 / 6), \lambda=2, k=1, z_{0}=2$.

$$
\phi(z)=\ln \left(\frac{-\sqrt{\left(4 \mu-\delta^{2}\right)} \tan \left(\left(\sqrt{\left(4 \mu-\delta^{2}\right)} / 2\right)(z+c)-\delta\right)}{2 \mu}\right)
$$

$$
\phi(z)=\ln \left(\frac{-\sqrt{\left(4 \mu-\delta^{2}\right)} \cot \left(\left(\sqrt{\left(4 \mu-\delta^{2}\right)} / 2\right)(z+c)-\delta\right)}{2 \mu}\right) .
$$

If $\delta^{2}-4 \mu<0, \mu=0, \delta \neq 0$, then

$$
\phi(z)=-\ln \left(\frac{\delta}{\exp (\delta(z+c)-1)}\right) .
$$

If $\delta^{2}-4 \mu=0, \mu \neq 0, \delta \neq 0$, then

$$
\phi(z)=\ln \left(-\frac{2(\delta(z+c)+2)}{\delta^{2}(z+c)}\right) .
$$

If $\delta^{2}-4 \mu=0, \mu=0, \delta=0$, then

$$
\phi(z)=\ln (z+c) .
$$

In the above equation, $C_{n} \neq 0, \delta$, and $\mu$ are constants and will be determined later and $c$ is an arbitrary constant. We consider the homogeneous balance between nonlinear terms and highest order derivatives of equation (27), and then we can get the positive integer $n$.

Step 3. Substitute equation (28) into (27) and accounting for the function $\exp (-\phi(z))$, we obtain a polynomial of $\exp (-\phi(z))$. We calculate all the coefficients of the same power of $\exp (-\phi(z))$ to zero and then we get a set of algebraic equations. By solving the algebraic equations, we obtain the values of $C_{n} \neq 0, \delta, \mu$; by putting these into equation (16) along with equations (30)-(36), we can get the determination of the solutions of equation (26).

Theorem 2. By employing the expexp $(-\phi(z))$-expansion method, we found that there will be three forms of solutions of (3).

$$
\begin{aligned}
& \text { If } \delta^{2}-4 \mu>0, \mu \neq 0, \\
& w_{11}=\frac{\sqrt{6 \beta \lambda} \delta k}{2}-\frac{2 \sqrt{6 \beta \lambda} k a \mu}{\sqrt{\delta^{2}-4 \mu} \tanh \left(\left(\left((z+c) \sqrt{\delta^{2}-4 \mu}\right) / 2\right)-\delta\right)} .
\end{aligned}
$$

In order to better describe $w_{11}(z)$, we make a graph of the function solution under specific parameters, as shown in Figure 3 in Section 7. Also,

$$
\begin{aligned}
& w_{12}=\frac{\sqrt{6 \beta \lambda} \delta k}{2}-\frac{2 \sqrt{6 \beta \lambda} k \mu}{\sqrt{\delta^{2}-4 \mu} \operatorname{coth}\left(\left(\left((z+c) \sqrt{\delta^{2}-4 \mu}\right) / 2\right)-\delta\right)}, \\
& w_{21}=-\frac{\sqrt{6 \beta \lambda} \delta k}{2}+\frac{2 \sqrt{6 \beta \lambda} k \mu}{\sqrt{\delta^{2}-4 \mu} \tanh \left(\left(\left((z+c) \sqrt{\delta^{2}-4 \mu}\right) / 2\right)-\delta\right)}, \\
& w_{22}=-\frac{\sqrt{6 \beta \lambda} \delta k}{2}+\frac{2 \sqrt{6 \beta \lambda} k \mu}{\sqrt{\delta^{2}-4 \mu} \operatorname{coth}\left(\left(\left((z+c) \sqrt{\delta^{2}-4 \mu}\right) / 2\right)-\delta\right)} .
\end{aligned}
$$

$$
\begin{aligned}
& \text { If } \delta^{2}-4 \mu<0, \mu \neq 0, \\
& w_{13}=\frac{\sqrt{6 \beta \lambda} \delta k}{2}-\frac{2 \sqrt{6 \beta \lambda} k \mu}{\sqrt{4 \mu-\delta^{2}} \tan \left(\left(\left((z+c) \sqrt{4 \mu-\delta^{2}}\right) / 2\right)-\delta\right)},
\end{aligned}
$$

In order to better describe $w_{13}(z)$, we make a graph of the function solution under specific parameters, as shown in Figure 4 in Section 7. Also, 


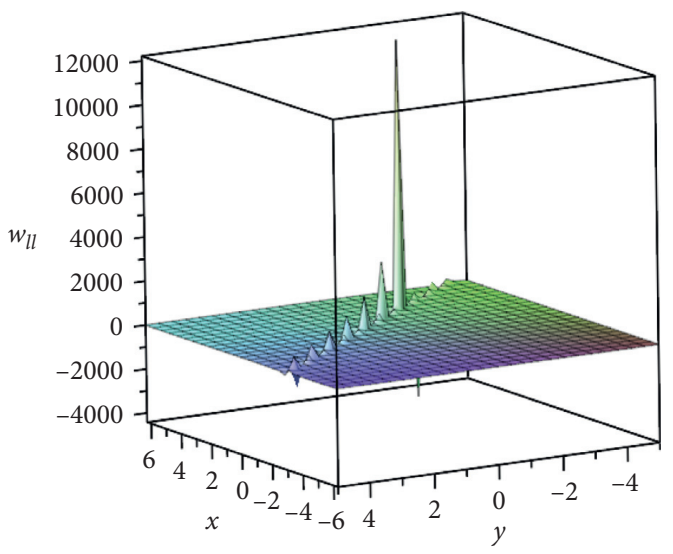

FIGURE 3: The solution of mBBM equation corresponding to $w_{11}(z)$, take $\beta=1, \lambda=1, \delta=4, \mu=3, k=1, a=1, c=2$.

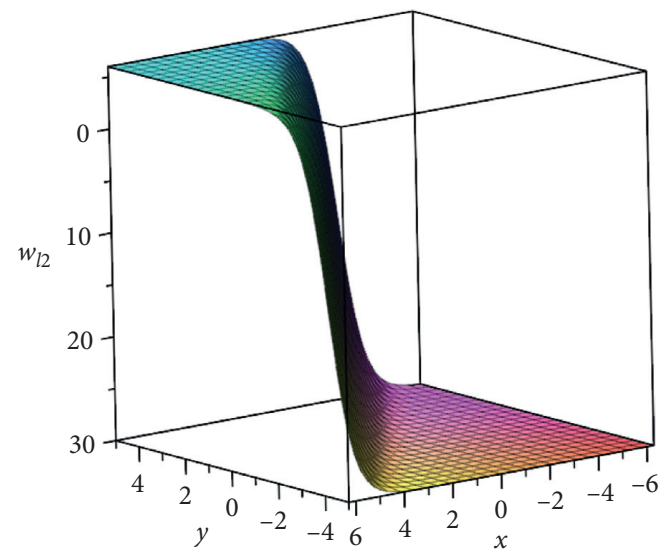

Figure 4: The solution of mBBM equation corresponding to $w_{12}(z)$, take $\beta=1, \lambda=1, \delta=4, \mu=3, k=1, a=1, c=2$.

$$
\begin{aligned}
& w_{14}=\frac{\sqrt{6 \beta \lambda} \delta k}{2}-\frac{2 \sqrt{6 \beta \lambda} k \mu}{\sqrt{4 \mu-\delta^{2}} \cot \left(\left(\left((z+c) \sqrt{4 \mu-\delta^{2}}\right) / 2\right)-\delta\right)}, \\
& w_{23}=-\frac{\sqrt{6 \beta \lambda} \delta k}{2}+\frac{2 \sqrt{6 \beta \lambda} k \mu}{\sqrt{4 \mu-\delta^{2}} \tan \left(\left(\left((z+c) \sqrt{4 \mu-\delta^{2}}\right) / 2\right)-\delta\right)}, \\
& w_{24}=-\frac{\sqrt{6 \beta \lambda} \delta k}{2}+\frac{2 \sqrt{6 \beta \lambda} k \mu}{\sqrt{4 \mu-\delta^{2}} \cot \left(\left(\left((z+c) \sqrt{4 \mu-\delta^{2}}\right) / 2\right)-\delta\right)} .
\end{aligned}
$$

If $\delta^{2}-4 \mu>0, \mu=0, \delta \neq 0$,

$$
\begin{aligned}
& w_{15}=\frac{\sqrt{6 \beta \lambda} \delta k}{2}+\frac{\sqrt{6 \beta \lambda} k \delta}{e^{\delta(z+c)-1}} \\
& w_{25}=-\frac{\sqrt{6 \beta \lambda} \delta k}{2}-\frac{\sqrt{6 \beta \lambda} k \delta}{e^{\delta(z+c)-1}} .
\end{aligned}
$$

If $\delta^{2}-4 \mu=0, \mu \neq 0, \delta \neq 0$,

$$
\begin{gathered}
w_{16}=\frac{\sqrt{6 \beta \lambda} \delta k}{2}-\frac{\sqrt{6 \beta \lambda} k \delta^{2}(z+c)}{2 \delta(z+c)+4}, \\
w_{26}=-\frac{\sqrt{6 \beta \lambda} \delta k}{2}+\frac{\sqrt{6 \beta \lambda} k \delta^{2}(z+c)}{2 \delta(z+c)+4} . \\
\text { If } \delta^{2}-4 \mu=0, \mu=0, \delta=0, \\
w_{17}=\frac{\sqrt{6 \beta \lambda} k}{(z+c)}, \\
w_{27}=-\frac{\sqrt{6 \beta \lambda} k}{(z+c)} .
\end{gathered}
$$

\section{Introduction of Sine-Cosine Method and Main Result}

On the other hand, the sine-cosine method was proved to be effective in handing problems where compactions are generated. In what follows, we simply describe this method because details can be found in [38].

The sine-cosine method admits the use of the solution in the form

$$
u(x, t)=\left\{\lambda_{1} \cos ^{\beta_{1}}(\mu z)\right\}, \quad|z|<\frac{\pi}{2 \mu},
$$

or in the form

$$
u(x, t)=\left\{\lambda_{1} \cos ^{\beta_{1}}(\mu z)\right\}, \quad|z|<\frac{\pi}{\mu},
$$

and zero otherwise. The parameters $\lambda_{1}, \mu$, and $\beta_{1}$ will be determined.

In equation (26), $P$ is a polynomial with an unknown function $\mu(x, t)$ and its derivatives in which nonlinear terms and highest order derivatives are involved. And it can be processed as follows:

Step 1. Insert the traveling wave transform $\mu(x, t)=$ $w(z), z=k(x-\lambda t)$ into equation (26) alternating it to the following ordinary differential equation (27). In equation (27), $K$ is a polynomial of $w(z)$ and its derivatives.

Step 2. Regarding that equation (27) has the following traveling wave solution:

$$
w(z)=\left\{\lambda_{1} \cos ^{\beta_{1}}(\mu z)\right\}, \quad|z|<\frac{\pi}{2 \mu},
$$

(i) or in the form

$$
w(z)=\left\{\lambda_{1} \cos ^{\beta_{1}}(\mu z)\right\}, \quad|z|<\frac{\pi}{\mu},
$$

and zero otherwise in equation (27). The parameters $\lambda_{1}, \mu$, and $\beta_{1}$ will be determined.

Step 3. Calculate all the coefficients of the same power of sine or cosine to zero and then we get a set of algebraic equations. By solving the algebraic 
equations, we obtain the values of $\lambda_{1}, \mu$, and $\beta_{1}$; we can get the determination of the solutions of equation (26).

Theorem 3. If we employ sine-cosine method, then all meromorphic solutions $w$ of equation (3) have the following forms of solutions:

(1) By using the sine method, we obtain the solution of (3) as follows:

$$
w_{T 1}(z)= \pm i \sqrt{6-6 \lambda} \csc \left(\frac{\sqrt{\lambda-1}}{k \sqrt{\beta \lambda}} z\right) .
$$

In order to better describe $w_{T 1}(z)$, we make a graph of the function solution under specific parameters, as shown in Figure 5 in Section 7 or

$$
w_{T 2}(z)= \pm i \sqrt{6-6 \lambda} \csc \left(\frac{-\sqrt{\lambda-1}}{k \sqrt{\beta \lambda}} z\right) .
$$

(2) By using the cosine method, we obtain the solution of (3) as follows:

$$
w_{T 3}(z)= \pm i \sqrt{6-6 \lambda} \sec \left(\frac{\sqrt{\lambda-1}}{k \sqrt{\beta \lambda}} z\right) .
$$

In order to better describe $w_{\mathrm{T} 3}(z)$, we make a graph of the function solution under specific parameters, as shown in Figure 6 in Section 7 or

$$
w_{T 4}(z)= \pm i \sqrt{6-6 \lambda} \sec \left(\frac{-\sqrt{\lambda-1}}{k \sqrt{\beta \lambda}} z\right) \text {. }
$$

\section{Proof of Theorems}

5.1. Proof of Theorem 1. Noting that our hypothesis $\left(\left(\beta \lambda k^{2}\right) / 3\right) \neq 0$ and putting (7) into (3), we have $q=1, p=2$, $c_{-1}= \pm \sqrt{6 \beta \lambda k^{2}}, c_{0}=0, c_{1}=\mp\left((\lambda-1) / \sqrt{6 \beta \lambda k^{2}}\right), c_{2}=(-b /$ $\left.\left(\beta \lambda k^{2}+4\right)\right), \quad c_{4}=\left(((1-\lambda) b) /\left(24 \beta^{2} \lambda^{2} k^{4}\right)\right)$, where $c_{3}$ is arbitrary.

Hence, (3) satisfies weak $\langle 1,2\rangle$ condition and is a 2order Briot-Bouquet differential equation. Obviously, (3) satisfies the dominant condition. By Lemma 1, we know that all meromorphic solutions of (3) belong to $W$, and then we will give the forms of all meromorphic solutions of (3).

By (16), we infer the indeterminant rational solutions of (3) with pole at $z=0$ that

$$
R_{1}(z)=\frac{c_{11}}{z}+\frac{c_{12}}{z-z_{1}}+c_{10}
$$

Substituting $R_{1}(z)$ into (3), we obtain two classes; one is as follows:

$$
R_{1,1}(z)= \pm \sqrt{6 \beta \lambda k^{2}} \frac{1}{z}
$$

where $\lambda=1$ and $b=0$. The other is

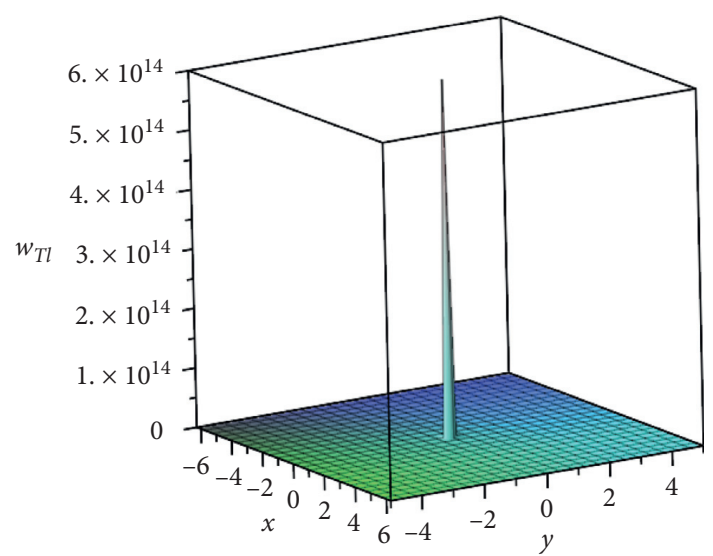

Figure 5: The solution of mBBM equation corresponding to $w_{T 1}(z)$, take $\lambda=2, \beta=3, k=1$.

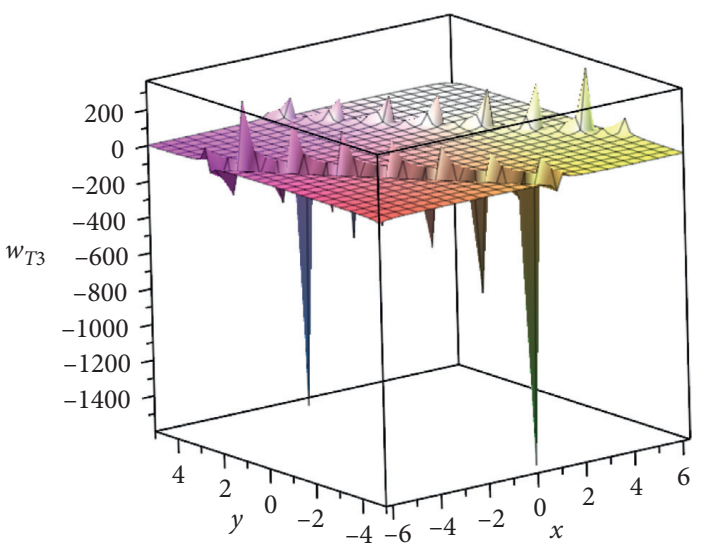

Figure 6: The solution of $\mathrm{mBBM}$ equation corresponding to $w_{T 3}(z)$, take $\lambda=2, \beta=3, k=1$.

$$
R_{1,2}(z)= \pm \sqrt{-6 \beta \lambda k^{2} \frac{1}{z_{1}^{2}}}\left(\frac{z_{1}}{z}-\frac{z_{1}}{z-z_{1}}-1\right)
$$

where $\lambda-1=\left(\left(6 \beta \lambda k^{2}\right) / z_{1}^{2}\right)$ and $b= \pm(2 / 3)\left(\left(6 \beta \lambda k^{2}\right) / z_{1}^{2}\right)^{(3 / 2)}$.

So, all rational solutions of (3) are as follows:

$$
\begin{aligned}
& w_{r, 1}(z)= \pm \sqrt{6 \beta \lambda k^{2}} \frac{1}{z-z_{0}}, \\
& w_{r, 2}(z)= \pm \sqrt{\frac{6 \beta \lambda k^{2}}{z_{1}^{2}}}\left(\frac{z_{1}}{z-z_{0}}-\frac{z_{1}}{z-z_{0}-z_{1}}-1\right),
\end{aligned}
$$

where $z_{0} \in \mathbb{C}, \lambda=1, b=0$ in the former formula, or given $z_{1} \neq 0, \lambda-1=\left(\left(6 \beta \lambda k^{2}\right) / z_{1}^{2}\right), b= \pm(2 / 3)\left(\left(6 \beta \lambda k^{2}\right) / z_{1}^{2}\right)^{(3 / 2)}$.

In order to have simply periodic solutions, set $\xi=\exp (\alpha z)$, put $w=R(\xi)$ into (3); then,

$$
A \alpha^{2}\left[\xi R^{\prime}+\xi^{2} R^{\prime \prime}\right]+B R+C R^{3}+D=0 .
$$

Putting 


$$
R_{2}(\xi)=\frac{c_{21}}{\xi-1}+\frac{c_{22}}{\xi-\xi_{1}}+c_{20}
$$

into (57), we get that

$$
\begin{aligned}
& R_{2,1}(\xi)=\frac{\alpha}{1-\xi_{1}} \sqrt{\frac{3}{2} \beta \lambda k^{2}} \\
& R_{2,2}(\xi)=\alpha \sqrt{\frac{3}{2} \beta \lambda k^{2}}\left(\frac{2}{\xi-1}+1\right) .
\end{aligned}
$$

Here,

$$
\begin{aligned}
\lambda-1 & =\left(\frac{1}{2}+\frac{6 \xi_{1}}{\left(1-\xi_{1}\right)^{2}}\right) \beta \lambda k^{2}, \\
-b & =\frac{8 C \xi_{1}\left(1+\xi_{1}\right) \alpha^{3}}{\left(1-\xi_{1}\right)^{3}}\left(\frac{3}{2} \beta \lambda k^{2}\right)^{(3 / 2)},
\end{aligned}
$$

in the former formula, or $\lambda-1=\left(\left(\beta \lambda k^{2} \alpha^{2}\right) / 2\right), b=0$.

Substituting $\xi=e^{\alpha z}$ into the above relation, we obtain simply periodic solutions of (3) with pole at $z=0$

$$
\begin{aligned}
& w_{s 0,1}(z)=\alpha \sqrt{\frac{3}{2} \beta \lambda k^{2}}\left(\operatorname{coth} \frac{\alpha}{2} z-\operatorname{coth} \frac{\alpha}{2}\left(z-z_{1}\right)-\operatorname{coth} \frac{\alpha}{2} z_{1}\right), \\
& w_{s 0,2}(z)=\alpha \sqrt{\frac{3}{2} \beta \lambda k^{2}}\left(\tanh \frac{\alpha}{2} z\right) .
\end{aligned}
$$

So, all simply periodic solutions of (3) are obtained by

$$
\begin{aligned}
& w_{s, 1}(z)=\alpha \sqrt{\frac{3}{2} \beta \lambda k^{2}}\left(\operatorname{coth} \frac{\alpha}{2}\left(z-z_{0}\right)-\operatorname{coth} \frac{\alpha}{2}\left(z-z_{0}-z_{1}\right)-\operatorname{coth} \frac{\alpha}{2} z_{1}\right), \\
& w_{s, 2}(z)=\alpha \sqrt{\frac{3}{2} \beta \lambda k^{2}}\left(\tanh \frac{\alpha}{2}\left(z-z_{0}\right)\right) .
\end{aligned}
$$

Here,

$$
\begin{aligned}
z_{0} & \in \mathbb{C}, \\
\lambda-1 & =\beta \lambda k^{2} \alpha^{2}\left(\frac{1}{2}+\frac{3}{2 \sinh ^{2}(\alpha / 2) z_{1}}\right), \\
b & =-\sqrt{\frac{3}{2} \beta \lambda k^{2}} \frac{\tanh (\alpha / 2) z_{1}}{\sinh ^{2}(\alpha / 2) z_{1}}, \quad z_{1} \neq 0,
\end{aligned}
$$

in the former formula, or $\lambda-1=\left(\left(\beta \lambda k^{2} \alpha^{2}\right) / 2\right), b=0$.

From (15) of Lemma 1 , we have indeterminant relations of elliptic solutions of (3) with pole at $z=0$ :

$$
w_{d 0}(z)=\frac{c_{-1}}{2} \frac{\wp^{\prime}(z)+F}{\wp(z)-E}+c_{30}
$$

where $F^{2}=4 E^{3}-g_{2} E-g_{2}$. Applying the conclusion II of Lemma 2 to $w_{d 0}(z)$, and noting the results of rational solutions obtained above, we deduce that $c_{30}=0, E=F=$ $0, g_{3}=0$. Then, we obtain that

$$
w_{d 0}(z)= \pm \frac{1}{2} \sqrt{6 \beta \lambda k^{2}} \frac{\wp^{\prime}(z)}{\wp(z)},
$$

where $g_{3}=0$. Therefore, all elliptic solutions of (3) are as follows:

$$
w_{d}(z)= \pm \frac{1}{2} \sqrt{6 \beta \lambda k^{2}} \frac{\wp^{\prime}(z)\left(z-z_{0}\right)}{\wp(z)\left(z-z_{0}\right)},
$$

where $z_{0} \in \mathbb{C}, g_{3}=0$. By using of III of Lemma 2, we rewrite it to the form

$$
w_{d}(z)= \pm \frac{1}{2} \sqrt{6 \beta \lambda k^{2}} \frac{(-\wp+c)\left(4 \wp c^{2}+4 \wp^{2} c+2 \wp^{\prime} d-\wp g_{2}-c g_{2}\right)}{\left(\left(12 c^{2}-g_{2}\right) \wp+4 c^{3}-3 c g_{2}\right) \wp^{\prime}+\left(4 \wp^{3}+12 c \wp^{2}-3 g_{2} \wp-c g_{2}\right) d^{\prime}}
$$

where $g_{3}=0, d^{2}=4 c^{3}-g_{2} c, g_{2}$, and $c$ are arbitrary.

5.2. Proof of Theorem 2. Taking the homogeneous balance between $w^{\prime \prime}$ and $w^{3}$ in (3), we obtain

$$
w(z)=C_{0}+C_{1} \exp (-\phi(z))
$$

where $C_{1} \neq 0, C_{0}$ are constants which need to be determined, and $\phi(z)$ satisfies equation $\phi^{\prime}(z)=\exp (-\phi(z))+\mu \exp$ $(\phi(z))+\delta$; here $\delta$ and $\mu$ are arbitrary constants.
From equation (68), we insert $w, w^{3}, w^{\prime \prime}$ into (3) and sort out the coefficients of $\exp (-\phi(z))$ to zero, so we get

$$
\begin{aligned}
& C_{1}= \pm \sqrt{6 \beta \lambda} k, \\
& C_{0}= \pm \frac{\sqrt{6 \beta \lambda} \delta k}{2},
\end{aligned}
$$

where $\delta= \pm\left(\sqrt{2 \beta \lambda\left(2 \beta \lambda k^{2} \mu+\lambda-1\right)} / \beta \lambda k\right)$ and $b=0$.

By substituting equation (69) into (68), we obtain 


$$
w_{1}(z)=\frac{\sqrt{6 \beta \lambda} \delta k}{2}+\sqrt{6 \beta \lambda} k e^{-\phi(z)},
$$

or

$$
w_{2}(z)=-\frac{\sqrt{6 \beta \lambda} \delta k}{2}-\sqrt{6 \beta \lambda} k e^{-\phi(z)} .
$$

We apply equations (30)-(36) into (70) and (71), respectively, to obtain traveling wave solutions of the $\mathrm{mBBM}$ equation as follows.

If $\delta^{2}-4 \mu>0, \mu \neq 0$,

$$
\begin{aligned}
& w_{11}=\frac{\sqrt{6 \beta \lambda} \delta k}{2}-\frac{2 \sqrt{6 \beta \lambda} k \mu}{\sqrt{\delta^{2}-4 \mu} \tanh \left(\left(\left((z+c) \sqrt{\delta^{2}-4 \mu}\right) / 2\right)-\delta\right)}, \\
& w_{12}=\frac{\sqrt{6 \beta \lambda} \delta k}{2}-\frac{2 \sqrt{6 \beta \lambda} k \mu}{\sqrt{\delta^{2}-4 \mu} \operatorname{coth}\left(\left(\left((z+c) \sqrt{\delta^{2}-4 \mu}\right) / 2\right)-\delta\right)}, \\
& w_{21}=-\frac{\sqrt{6 \beta \lambda} \delta k}{2}+\frac{2 \sqrt{6 \beta \lambda} k \mu}{\sqrt{\delta^{2}-4 \mu} \tanh \left(\left(\left((z+c) \sqrt{\delta^{2}-4 \mu}\right) / 2\right)-\delta\right)}, \\
& w_{22}=-\frac{\sqrt{6 \beta \lambda} \delta k}{2}+\frac{2 \sqrt{6 \beta \lambda} k \mu}{\sqrt{\delta^{2}-4 \mu} \operatorname{coth}\left(\left(\left((z+c) \sqrt{\delta^{2}-4 \mu}\right) / 2\right)-\delta\right)} .
\end{aligned}
$$

If $\delta^{2}-4 \mu<0, \mu \neq 0$,

$$
\begin{aligned}
& w_{13}=\frac{\sqrt{6 \beta \lambda} \delta k}{2}-\frac{2 \sqrt{6 \beta \lambda} k \mu}{\sqrt{4 \mu-\delta^{2}} \tan \left(\left(\left((z+c) \sqrt{4 \mu-\delta^{2}}\right) / 2\right)-\delta\right)}, \\
& w_{14}=\frac{\sqrt{6 \beta \lambda} \delta k}{2}-\frac{2 \sqrt{6 \beta \lambda} k \mu}{\sqrt{4 \mu-\delta^{2}} \cot \left(\left(\left((z+c) \sqrt{4 \mu-\delta^{2}}\right) / 2\right)-\delta\right)}, \\
& w_{23}=-\frac{\sqrt{6 \beta \lambda} \delta k}{2}+\frac{2 \sqrt{6 \beta \lambda} k \mu}{\sqrt{4 \mu-\delta^{2}} \tan \left(\left(\left((z+c) \sqrt{4 \mu-\delta^{2}}\right) / 2\right)-\delta\right)}, \\
& w_{24}=-\frac{\sqrt{6 \beta \lambda} \delta k}{2}+\frac{2 \sqrt{6 \beta \lambda} k \mu}{\sqrt{4 \mu-\delta^{2}} \cot \left(\left(\left((z+c) \sqrt{4 \mu-\delta^{2}}\right) / 2\right)-\delta\right)} .
\end{aligned}
$$

If $\delta^{2}-4 \mu>0, \mu=0, \delta \neq 0$,

$$
\begin{aligned}
& w_{15}=\frac{\sqrt{6 \beta \lambda} \delta k}{2}+\frac{\sqrt{6 \beta \lambda} k \delta}{e^{\delta(z+c)-1}}, \\
& w_{25}=-\frac{\sqrt{6 \beta \lambda} \delta k}{2}-\frac{\sqrt{6 \beta \lambda} k \delta}{e^{\delta(z+c)-1}} .
\end{aligned}
$$

If $\delta^{2}-4 \mu=0, \mu \neq 0, \delta \neq 0$,

$$
\begin{aligned}
& w_{16}=\frac{\sqrt{6 \beta \lambda} \delta k}{2}-\frac{\sqrt{6 \beta \lambda} k \delta^{2}(z+c)}{2 \delta(z+c)+4}, \\
& w_{26}=-\frac{\sqrt{6 \beta \lambda} \delta k}{2}+\frac{\sqrt{6 \beta \lambda} k \delta^{2}(z+c)}{2 \delta(z+c)+4} .
\end{aligned}
$$

If $\delta^{2}-4 \mu=0, \mu=0, \delta=0$,

$$
\begin{aligned}
& w_{17}=\frac{\sqrt{6 \beta \lambda} k}{(z+c)} \\
& w_{27}=-\frac{\sqrt{6 \beta \lambda} k}{(z+c)} .
\end{aligned}
$$

5.3. Proof of Theorem 3. The sine-cosine method admits the use of the solution in the form

$$
w(z)=\left\{\lambda_{1} \cos ^{\beta_{1}}(\mu z)\right\}, \quad|z|<\frac{\pi}{2 \mu},
$$

or in the form 


$$
w(z)=\left\{\lambda_{1} \sin ^{\beta_{1}}(\mu z)\right\}, \quad|z|<\frac{\pi}{\mu}
$$

and zero otherwise. The parameters $\lambda_{1}, \mu, \beta_{1}$ will be determined. We substitute

$$
w(z)=\left\{\lambda_{1} \cos ^{\beta_{1}}(\mu z)\right\}
$$

into (3) and obtain

$$
\begin{aligned}
& \left(\lambda \beta k^{2} \beta_{1}^{2} \lambda_{1} \mu^{2}-\lambda \beta k^{2} \beta_{1} \lambda_{1} \mu^{2}\right) \cos ^{\left(\beta_{1}-2\right)}(\mu z) \\
& +\left(\lambda \lambda_{1}-\lambda_{1}-\lambda \beta k^{2} \beta_{1}^{2} \lambda_{1} \mu^{2}\right) \cos ^{\beta_{1}}(\mu z) \\
& -\frac{1}{3} \lambda_{1}^{3} \cos ^{3 \beta_{1}}(\mu z)-b=0 .
\end{aligned}
$$

Collecting the coefficients of each pair of cosine functions of the same exponent and setting it equal to zero, we obtain the following system of algebraic equations:

$$
\begin{aligned}
\beta_{1}-1 & \neq 0, \\
\beta_{1}-2 & =3 \beta_{1}, \\
k^{2} \beta \beta_{1}^{2} \lambda \lambda_{1} \mu^{2}-k^{2} \beta \beta_{1} \lambda \lambda_{1} \mu^{2} & =\frac{1}{3} \lambda_{1}^{3}, \\
\lambda \lambda_{1}-\lambda_{1} & =k^{2} \beta \beta_{1}^{2} \lambda \lambda_{1} \mu^{2} .
\end{aligned}
$$

Solving this system gives

$$
\begin{aligned}
\beta_{1} & =-1, \\
\mu & = \pm \frac{\sqrt{\lambda-1}}{k \sqrt{\beta \lambda}} \\
\lambda_{1} & = \pm i \sqrt{6-6 \lambda} .
\end{aligned}
$$

At last, we get

$$
w_{c 1}(z)= \pm i \sqrt{6-6 \lambda} \sec \left(\frac{\sqrt{\lambda-1}}{k \sqrt{\beta \lambda}} z\right)
$$

or

$$
w_{c 2}(z)= \pm i \sqrt{6-6 \lambda} \sec \left(\frac{-\sqrt{\lambda-1}}{k \sqrt{\beta \lambda}} z\right) .
$$

We substitute

$$
w(z)=\left\{\lambda_{1} \sin ^{\beta_{1}}(\mu z)\right\}
$$

into (3) and obtain

$$
\begin{aligned}
& \left(\lambda \beta k^{2} \beta_{1}^{2} \lambda_{1} \mu^{2}-\lambda \beta k^{2} \beta_{1} \lambda_{1} \mu^{2}\right) \sin ^{\left(\beta_{1}-2\right)}(\mu z) \\
& +\left(\lambda \lambda_{1}-\lambda_{1}-\lambda \beta k^{2} \beta_{1}^{2} \lambda_{1} \mu^{2}\right) \sin ^{\beta_{1}}(\mu z) \\
& -\frac{1}{3} \lambda_{1}^{3} \sin ^{3 \beta_{1}}(\mu z)-b=0 .
\end{aligned}
$$

Collecting the coefficients of each pair of cosine functions of the same exponent and setting it equal to zero, we obtain the following system of algebraic equations:

$$
\begin{aligned}
\beta_{1}-1 & \neq 0, \\
\beta_{1}-2 & =3 \beta_{1}, \\
k^{2} \beta \beta_{1}^{2} \lambda \lambda_{1} \mu^{2}-k^{2} \beta \beta_{1} \lambda \lambda_{1} \mu^{2} & =\frac{1}{3} \lambda_{1}^{3}, \\
\lambda \lambda_{1}-\lambda_{1} & =k^{2} \beta \beta_{1}^{2} \lambda \lambda_{1} \mu^{2} .
\end{aligned}
$$

Solving this system gives

$$
\begin{aligned}
& \beta_{1}=-1, \\
& \mu= \pm \frac{\sqrt{\lambda-1}}{k \sqrt{\beta \lambda}} \\
& \lambda_{1}= \pm i \sqrt{6-6 \lambda} .
\end{aligned}
$$

At last, we obtain

$$
w_{c 1}(z)= \pm i \sqrt{6-6 \lambda} \csc \left(\frac{\sqrt{\lambda-1}}{k \sqrt{\beta \lambda}} z\right),
$$

or

$$
w_{c 2}(z)= \pm i \sqrt{6-6 \lambda} \csc \left(\frac{-\sqrt{\lambda-1}}{k \sqrt{\beta \lambda}} z\right)
$$

\section{Comparison}

Implementing the $\exp (-\phi(z))$-expansion method, we found that there are fourteen forms of solutions of $\mathrm{mBBM}$ equation. Using the complex method, we found eight solutions of mBBM equation. In the above two methods, the solutions of equations (76), (77), and (55) are the same. Finally, using sine-cosine method, we found eight styles' solutions of mBBM equation. From the above results, we can find more solutions by the $\exp (-\phi(z))$-expansion method, whereas we can obtain elliptic function solutions just by the complex method. Also, we know that, by using the $\exp (-\phi(z))$-expansion method and complex method, we find the hyperbolic functions solutions for the $\mathrm{mBBM}$ equation. By using the sine-cosine method, we only find the trigonometric function solutions for $\mathrm{mBBM}$ equation. Each of three methods has its own characteristics. We firmly believe that the complex method presented in this paper can be more effectively applied to seek solutions of other nonlinear evolution equations now and in the future. These three methods are very useful tools for finding the exact solutions of nonlinear evolution equations.

\section{Computer Simulations}

In this section, we illustrate some results through computer simulations. We carry out further analysis to the properties of simply periodic solutions $w_{s, 1}(z), w_{11}(z), w_{T 1}(z), w_{r, 1}(z)$, $w_{12}(z), w_{T 3}(z)$ of the mBBM equation in the figures.

From Figures 1 and 2, we can obtain the dynamic behavior of solving $w_{s, 1}(z), w_{11}(z)$. From Figure 3 , the lump solution $w_{T 1}(z)$ has one global maximum point. 
From Figure $4, w_{r, 1}(z)$ represents the singular soliton for the special parameters. From Figure 5, we can get the continuous dynamic behavior of solving $w_{12}(z)$. From Figure $6, w_{T 3}(z)$ has many distinct generation poles.

\section{Conclusions}

Complex method is very effective tool for seeking the exact solutions of nonlinear evolution equations, and equation (3) is one of most important auxiliary equations because many nonlinear evolution equations can be transformed into it. In this paper, we employed the complex method, $\exp (-\phi(z))$ expansion method, and sine-cosine method to get the exact solutions of modified BBM equation. In this way, we can reduce the dimension of the nonlinear evolution equations related to engineering and mathematical physics. We obtain twenty-eight forms of solutions of mBBM equation. It shows these methods are very efficient and powerful in solving the exact solutions of nonlinear evolution equations now and in the future. We can apply the idea of this research to other nonlinear evolution equations. Our work shows that there exist some classes of rational solutions $w_{r, 1}(z), w_{r, 2}(z)$ and simple periodic solutions $w_{s, 1}(z), w_{s, 2}(z)$ which are not degenerated successively by the elliptic function solutions and are new.

\section{Data Availability}

No data were used to support this study.

\section{Conflicts of Interest}

The authors declare that they have no conflicts of interest.

\section{Authors' Contributions}

All authors typed, read, and approved the final manuscript.

\section{Acknowledgments}

This work was supported by the Plateau Disciplines in Shanghai. Also, this work was supported by Leading Academic Applied Mathematical of Shanghai Dianji University (16JCXK02), Humanity and Social Science Youth Foundation of Ministry of Education (18YJC630120), Shanghai Multi Direction Die Forging Engineering Technology Research Center (No. 20DZ2253200), National Key R\&D Program-Research on Modernization of Traditional Chinese Medicine (2018YFC1707802), and Science and Technology Support Project in Biomedical Field of "Science and Technology Innovation Action Plan" of Shanghai in 2019 (No. 19441914900).

\section{References}

[1] D. K. Campbell and Y. N. Huang, "Nonlinear science from sample to practical," Mechanical Progress, vol. 19, no. 4, pp. 90-100, 1989.

[2] C. Z. Tian, L. L. Wang, and Y. Yang, "Distribution network evaluation method combined with nonlinear mapping and clustering analysis," Journal of Power Systems and Automation, vol. 30, no. 12, pp. 145-150, 2018.

[3] L. Jose, D. S. Pinto, and L. Miguel, "On human life risk-assessment and sensitive ground fault protection in MV distribution networks," IEEE Transactions on Power Delivery, vol. 25, no. 4, pp. 2319-2327, 2010.

[4] C. S. Liu and L. C. Jiao, "Application of nonlinear programming in reactive power compensation optimization of mine high-voltage distribution network," Journal of Coal, vol. 27, no. 4, pp. 444-448, 2002.

[5] J. J. Liang, F. Lan, Z. G. Nong, and J. H. Li, "Probability load flow of distribution network with considering wind power nonlinear correlation," Electric Power Construction, vol. 38, no. 7, pp. 34-43, 2017.

[6] W. J. Liu, J. M. Wang, and P. F. Wang, "Analysis and research on electrical characteristics of nonlinear load," Shan $X i$ Electric Power, vol. 44, no. 8, pp. 71-75, 2016.

[7] Z. P. Wang, X. G. Jiang, and Z. C. Zhang, "Relay protection in smart grid environment," Protection and Control of Power System, vol. 41, no. 2, pp. 13-18, 2013.

[8] P. H. Yang, W. Y. Liu, and Y. L. Wei, "Nonlinear coordinated control of thyristor controlled series compensation with excitation system of generator," Journal of Power System and Automation, vol. 26, no. 10, pp. 51-55, 2014.

[9] G. B. Xu and J. Wang, "Stability analysis of small random disturbances in nonlinear power systems," Power Grid Technology, vol. 38, no. 10, pp. 2735-2740, 2014.

[10] F. S. Wang, K. C. Zhang, and P. P. Shen, "A new mathematical model for the diagnosis of ground grid corrosion in power system and its simulation calculation," Journal of Applied Mathematics in Universities, vol. 22, no. 2, pp. 141-152, 2007.

[11] X. H. Lu and Y. T. Zheng, "Power system load forecasting model based on nonlinear time series analysis," Power and Energy, vol. 37, no. 2, pp. 197-201, 2015.

[12] T. Tokihiro, J. Satsuma, and R. Willox, "On special function solutions to nonlinear integrable equations," Physics Letters A, vol. 236, no. 1-2, pp. 23-29, 1997.

[13] S. K. Wang, H. Y. Guo, and K. Wu, "Back scattering transformation and normal Riemann-Hilbert problem," Acta Physica Sinica, vol. 32, no. 12, pp. 1589-1594, 1983.

[14] C. S. Gardner, J. M. Greene, M. D. Kruskal, and R. M. Miura, "Method for solving the Korteweg-devries equation," Physical Review Letters, vol. 19, no. 19, pp. 1095-1097, 1967.

[15] H. J. Li and H. Y. Ruan, "A new solution to the 2+1-dimensional KdV equation and the hirota-satsuma equation," Journal of Ningbo University, vol. 17, no. 2, pp. 125-129, 2004.

[16] M. Musette and R. Conte, "Riccati pseudopotential of AKNS two-family NLPDES by Painleve analysis," Theoretical and Mathematical Physics, vol. 99, no. 3, pp. 738-744, 1994.

[17] J.-H. He and X.-H. Wu, "Exp-function method for nonlinear wave equations," Chaos Solitons and Fractals, vol. 30, no. 3, pp. 700-708, 2006.

[18] M. M. A. Khater and D. Baleanu, "On abundant new solutions of two fractional complex models," Advances in Difference Equations, vol. 1, pp. 1-14, 2020.

[19] M. M. A. Khater, C. Park, D. Lu, and R. A. M. Attia, “Analytical, semi-analytical, and numerical solutions for the Cahn-Allen equation," Advances in Difference Equations, vol. 1, pp. 1-12, 2020.

[20] M. M. A. Khater, R. A. M. Attia, W. Alharbi, A. H. Abdel-Aty, and D. Lu, "Abundant analytical and numerical solutions of the fractional microbiological densities model in bacteria cells as a result of diffusion mechanisms," Chaos, Solitons and Fractals, vol. 136, 2020. 
[21] M. Gao, "An algebraic method to obtain exact traveling solutions of nonlinear differential equations," Journal of Minjiang University, vol. 27, no. 5, pp. 8-13, 2006.

[22] B. Gao and X. Li, "Envelope isolated wave solution of daveystewartson equation," Journal of Yuxi Normal University, vol. 26, no. 4, pp. 25-29, 2010.

[23] E. A. Saied, R. G. Abd El-Rahman, and M. I. Ghonamy, "A generalized Weierstrass elliptic function expansion method for solving some nonlinear partial differential equations," Computers and Mathematics with Applications, vol. 58, no. 9, pp. 1725-1735, 2009.

[24] R. Greger, "Nonlinear partial differential equations solved by projective riccati equations ansatz," Zeitschrift Für Naturforschung A, vol. 58, no. 9, pp. 511-519, 2003.

[25] Y. Yang, J.-m. Qi, X.-h. Tang, and Y.-y. Gu, "Further results about traveling wave exact solutions of the $(2+1)$-dimensional modified kdv equation," Advances in Mathematical Physics, vol. 2019, pp. 1-10, 2019.

[26] T. B. Benjamin, J. L. Bona, and J. J. Mahony, "Model equations for long waves in nonlinear dispersive systems," Philosophical Transactions of the Royal Society of London. Series A, Mathematical and Physical Sciences, vol. 272, no. 1220, pp. 47-78, 1972.

[27] A. Ganguly and A. Das, "Explicit solutions and stability analysis of the $(2+1)$ dimensional KP-BBM equation with dispersion effect," Communications in Nonlinear Science and Numerical Simulation, vol. 25, no. 1-3, pp. 102-117, 2015.

[28] W. G. Zhang and Z. S. Li, "A class of accurate traveling wave solutions for $\mathrm{n}$-dimensional B-BBM equations and B-Kdv equations," Journal of Applied Mathematics, vol. 6, no. 2, pp. 282-289, 1991.

[29] Y. D. Shang, "The exact solution of combined BBM and modified BBM equation," Journal of Basic Science of Textile University, vol. 11, no. 2, pp. 138-142, 1998.

[30] S. S. Liu, H. Chen, and Z. T. Fu, "The invariance of multi-stage exact solutions of Lamé function and nonlinear evolution equation," Acta Physica Sinica, vol. 52, no. 8, pp. 1842-1847, 2003.

[31] S. Tagetu and N. Serendorji, "New exact solitary wave solutions for BBM equation and modified BBM equation," Acta Physica Sinica, vol. 53, no. 12, pp. 4052-4060, 2004.

[32] Z. L. Liu, "A new analytic solution of mBBM equation," Practice and Recognition of Mathematics, vol. 43, no. 22, pp. 286-290, 2013.

[33] J. Yang and Q. J. Feng, "Applied the improved simple equation method to find the exact solution of the mBBM equation," Journal of Kaili University, vol. 35, no. 3, pp. 12-13, 2017.

[34] A. K. Gupta and J. Hazarika, "On the solitary wave solutions of modified Benjamin-Bona-Mahony equation for unidirectional propagation of long waves," Pramana, vol. 94, no. 1, pp. 1-8, 2020.

[35] W. J. Yuan, B. Xiao, Y. H. Wu, and J. M. Qi, “The general traveling wave solutions of the Fisher type equations and some related problems," Advances in Difference Equations, vol. 1, pp. 1-15, 2014.

[36] W. Yuan, F. Meng, Y. Huang, and Y. Wu, "All traveling wave exact solutions of the variant Boussinesq equations," Applied Mathematics and Computation, vol. 268, pp. 865-872, 2015.

[37] Y. Y. Gu and J. M. Qi, "Symmetry reduction and exact solutions of two higher-dimensional nonlinear evolution equations," Journal of Inequalities and Applications, vol. 1, pp. 1-19, 2017.
[38] A.-M. Wazwaz, "Compact and noncompact physical structures for the ZK-BBM equation," Applied Mathematics and Computation, vol. 169, no. 1, pp. 713-725, 2005.

[39] W. Yuan, W. Xiong, J. Lin, and Y. Wu, "All meromorphic solutions of an auxiliary ordinary differential equation and its applications," Acta Mathematica Scientia, vol. 35, no. 5, pp. 1241-1250, 2015.

[40] D. Z. Lü, "Abundant Jaccobi elliptic function solutions nonlinear evolution equations," Acta Physica Sinica, vol. 54, no. 10 , pp. 4501-4505, 2005.

[41] A. E. Eremenko, L. Liao, and T. W. Ng, "Meromorphic solutions of higher order Briot-Bouquet differential equations," Mathematical Proceedings of the Cambridge Philosophical Society, vol. 146, no. 1, pp. 197-206, 2009.

[42] W. J. Yuan, Z. F. Huang, M. Z. Fu, and J. C. Lai, "The general solutions of an auxiliary ordinary differential equation using complex method and its applications," Advances in Difference Equations, vol. 1, pp. 1-9, 2014.

[43] W. J. Yuan, Y. D. Shang, Y. Huang, and H. Wang, "The representation of meromorphic solutions to certain ordinary differential equations and its applications," Science in China: Mathematics, vol. 6, no. 43, pp. 563-575, 2013.

[44] N. A. Kudryashov, "Meromorphic solutions of nonlinear ordinary differential equations," Communications in Nonlinear Science and Numerical Simulation, vol. 10, no. 15, pp. 2778-2790, 2010.

[45] S. Lang, Elliptic Functions, pp. 5-21, Springer, New York, NY, USA, 1987.

[46] R. Conte and M. Musette, "Elliptic general analytic solutions," Studies in Applied Mathematics, vol. 1, no. 123, pp. 63-81, 2009. 